\title{
Comparison of short and long co-incubation time of gametes for in vitro fertilization
}

\section{Pallop Pongsuthirak*}

Department of Obstetrics and Gynecology, Buddhachinaraj Hospital Medical School, Phitsanulok, Thailand

Received: 18 September 2019

Accepted: 24 September 2019

\section{*Correspondence:}

Dr. Pallop Pongsuthirak,

E-mail: palloppong@yahoo.co.th

Copyright: () the author(s), publisher and licensee Medip Academy. This is an open-access article distributed under the terms of the Creative Commons Attribution Non-Commercial License, which permits unrestricted non-commercial use, distribution, and reproduction in any medium, provided the original work is properly cited.

\section{ABSTRACT}

Background: The short and long co-incubation time of gametes for in vitro fertilization are still debatable issues. This study aims to investigate the effects of short and long co-incubation time of gametes on fertilization, polyspermy, embryonic developmental potential, and clinical outcomes.

Methods: Sixty-five patients undergoing IVF treatment were invited to participate in the study between May 2017 and March 2019. Ovarian hyperstimulation was prescribed and oocytes were obtained by trans-vaginal aspiration under ultrasound guidance. Sibling oocytes were randomly allocated to short co-incubation for 4 hours (Group I) in 352 oocytes and long co-incubation for 16-18 hours in 363 oocytes (Group II). Rescue ICSI was carried out if total fertilization failure was documented. Fertilization, embryonic development, and pregnancy outcomes were determined.

Results: No significant differences between short and long co-incubation were found in fertilization, polyspermy, cleavage, blastocyst, implantation, clinical pregnancy, and live birth rates.

Conclusions: The present study showed that short co-incubation of gametes had no significant difference in fertilization, polyspermy, embryo development, and pregnancy outcomes when compared to long co-incubation. The short co-incubation with early cumulus cell removal and rescue ICSI may have the potential to help a couple who had total fertilization failure.

Keywords: Cumulus cell removal, In-vitro fertilization, Long co-incubation, Rescue ICSI, Short co-incubation, Total fertilization failure

\section{INTRODUCTION}

During conventional in vitro fertilization procedure, the cumulus-oocyte complexes are inseminated with sperms and co-incubated until 18-20 hours then the cumulus cells are removed to determine fertilization. The long exposure of oocyte to many sperms is considered to be suboptimal culture condition which can produce oxygen free radical that potentially causes zonal hardening and impairs embryonic development and implantation. ${ }^{1}$
Currently, short co-incubation time (1-4 hours) is widely used and claims to have better outcomes. ${ }^{2,3}$ Moreover, the additional benefit is early detection of total fertilization failure (TFF) after cumulus cell denudation and rescue intracytoplasmic sperm injection (R-ICSI) can be offered in such a situation with promising results. ${ }^{4-6}$ To date, there are still debatable issues on short and long coincubation time of gametes for in vitro fertilization. This study aims to investigate the effects of short and long coincubation time of gametes on fertilization, polyspermy, 
embryonic developmental potential, and clinical outcomes.

\section{METHODS}

The Research Ethics Committee of Buddhachinaraj Hospital Medical School approved this study (No.166/62) and Thai Clinical Trial Registration (TCTR20190916001). This prospective randomized clinical study was performed between May 2017 and March 2019. Patients undergoing IVF treatment were invited to participate in this study and signed written informed consent.

The inclusion criteria were women 20-38 years old, had at least 6 retrieved oocytes and normal semen parameters. The etiologies for infertility included tubal disease, endometriosis, ovulatory dysfunction, unexplained infertility. The oocytes were randomly allocated into two groups short co-incubation time (Group I) defined as cumulus-enclosed oocyte co-incubated with sperms for 4 hours and long co-incubation time (Group II) defined as cumulus-enclosed oocyte co-incubated with sperms for 16-18 hours.

Rescue ICSI was performed when total fertilization failure (TFF) was deemed to occur in the short coincubation group after 6 hours of insemination. Embryo transfers were selected randomly into two groups depending on whether the transferred embryos originated from short or long co-incubation time group.

\section{Controlled ovarian hyperstimulation and oocyte retrieval}

All patients were treated by controlled ovarian hyperstimulation $(\mathrm{COH})$. Briefly, gonadotropin-releasing hormone agonist was administered for down-regulation in the mid-luteal phase of the previous cycle. Folliclestimulating hormone and/or human menopausal gonadotropins in individually adapted doses were injected after pituitary desensitization. The patients then received 5000-10,000 IU of human chorionic gonadotropin (hCG) when at least three follicles were $\geq 18 \mathrm{~mm}$. Oocyte retrieval was performed under transvaginal ultrasound guidance at 36-38 hours later.

\section{Sperm preparation and insemination}

Semen samples were collected by masturbation in the morning of the oocyte retrieval day following 3-5 days of sexual abstinence. Sperm concentration, motility, and morphology were evaluated under a light microscope based on the World Health Organization criteria (WHO, 1992). Sperm preparation was performed using gradient centrifugation. The sperm pellet was then placed at the bottom of the fresh insemination medium and incubated at $37^{\circ} \mathrm{C}$ in $6 \% \mathrm{CO}_{2}$ incubator to facilitate the swim-up technique.
Active motile sperms were harvested for insemination containing $15,000-50,000$ sperms in $50 \mu \mathrm{L}$ of insemination medium.

\section{Cumulus cells removal}

Oocytes were transferred to new sperm-free insemination medium after 4hours of co-incubation. In Group I, cumulus cells was mechanically removed at 6hours postinsemination using a denuding pipette (Flexipet, Cook) inner diameter of $140 \mu \mathrm{m}$ under an inverted microscope. Fertilization was defined as the observation of a second polar body and total fertilization failure was defined as the absence of a second polar body in any of the mature oocytes. Rescue ICSI was performed if none of the oocytes have early fertilization after 6 hours of insemination. In Group II, the cumulus cells were removed at 16-18 hours after insemination.

\section{Fertilization assess and embryo culture}

Normal fertilization was defined as the presence of two pronuclei and polyspermy was defined as the presence of $\geq 3$ pronuclei. The developmental competence of zygotes was assessed after 96-120 hours. Embryos were placed in cleavage media during days 1-3 after fertilization and followed by blastocyst media during days 4-5. Embryo morphology was classified on day 5 . The blastocysts were assigned a score based on the Gardner system with the high-quality blastocysts having scores of $\geq 4 \mathrm{BB}$. The surplus high-scoring blastocysts were cryopreserved for future transfers.

\section{Clinical and birth outcomes}

Embryo transfer took place day 5 after oocytes retrieval under ultrasound guidance. The number of embryos was restricted to one blastocyst to reduce the risk of multiple gestations. Luteal support with progesterone was initiated on day 3 after oocyte retrieval to all patients. The implantation rate was defined as the number of gestational sacs divided by the number of embryos that were transferred. The fetal heartbeat was determined by ultrasonography to confirm clinical pregnancy at 5 weeks after embryo transfer. The details of the patient's ongoing pregnancy were recorded at week 20 and also birth outcome.

\section{Statistical analysis}

All analyses were performed using SPSS software (version 13.0, SPSS Inc., Chicago, IL). Continuous data were compared using Student's t-test and proportional data were compared using the $\chi 2$ test. P-value of $<0.05$ was considered the statistical significance.

Our sample size calculation showed that at least 245 oocytes in each arm would be required to demonstrate a $10 \%$ difference in maturation rates between oocytes in 
the two groups, given a type I error of 5\% (two-tailed) and a type II error of $20 \%$.

\section{RESULTS}

A total of 65 women were eligible in the study. Five patients were excluded due to total fertilization failure and rescue ICSI was carried out. The rest of 60 women were $32.4 \pm 2.3$ years old. Twenty-nine cases $(48.3 \%)$ had tubal factors, $6(10.0 \%)$ had endometriosis, $9(15.0 \%)$ had polycystic ovary syndrome and 16 (26.7\%) had unexplained infertility. Seven hundred and fifteen oocytes were randomly allocated to short co-incubation time (Group I) and long co-incubation time (Group II). Three hundred and fifty-two mature oocytes were in Group I and 363 oocytes were in Group II. There were no significant differences in all treatment parameters between the two groups. The details of fertilization and embryo development were summarized in Table 1. No significant differences were observed in fertilization, polyspermy, cleavage, and blastocyst rate (Table 1). The embryo transfers were conducted in 60 cycles, of which 32 cycles obtained the embryos from the Group I and 28 cycles from Group II. There were no significant differences between two groups in terms of age, the number of oocytes, implantation rates, clinical pregnancy, ongoing pregnancy, and live birth rate between the patients with embryos transferred from the short and the long co-incubation group (Table 2). There was also no significant difference in obstetric and prenatal outcomes in short co-incubation compared to long co-incubation group.

Table 1: Comparison of parameters between short and long co-incubation time groups.

\begin{tabular}{|llll|}
\hline & Short co-incubation (Group I) n (\%) & Long co-incubation (Group II) n (\%) & P-value* \\
\hline Mature oocytes & 352 & 363 & - \\
\hline Two pronuclei & $253(71.9 \%)$ & $255(70.2 \%)$ & 0.691 \\
\hline Polyspermy & $35(9.9 \%)$ & $25(6.9 \%)$ & 0.181 \\
\hline Cleavage & $227(89.7 \%)$ & $218(85.5 \%)$ & 0.369 \\
\hline Blastocyst formation & $127(55.9 \%)$ & $110(50.5 \%)$ & 0.333 \\
\hline High quality blastocyst & $76(59.8 \%)$ & $57(51.8 \%)$ & 0.237 \\
\hline
\end{tabular}

Table 2: Comparison of pregnancy outcomes between short and long co-incubation time groups.

\begin{tabular}{|llll|} 
& Short co-incubation (Group II) & Long co-incubation (Group II) & P-value* \\
\hline Patients (n) & 32 & 28 & - \\
\hline Implantation rate n (\%) & $12 / 32(37.5 \%)$ & $9 / 28(32.1 \%)$ & 0.871 \\
\hline Clinical pregnancy rate n (\%) & $11 / 32(34.4 \%)$ & $8 / 28(28.6 \%)$ & 0.838 \\
\hline Ongoing pregnancy rate n (\%) & $10(31.3 \%)$ & $8(28.6 \%)$ & 0.955 \\
\hline Live birth rate $\mathrm{n}(\%)$ & $10(31.3 \%)$ & $8(28.6 \%)$ & 0.955 \\
\hline
\end{tabular}

$* \chi^{2}$-tests

Interestingly, there were 5 patients whose oocytes had short co-incubation and documented as total fertilization failure. Rescue ICSI was performed. Twenty-three of 30 oocytes $(76.7 \%)$ had normal fertilization without the detection of polyspermy and 3 embryos were transferred in 3 cycles resulting in one single pregnancy with an uneventful course of pregnancy and delivered a normal newborn. However, in the long co-incubation group, 2 patients had simultaneous fertilization in the sibling oocytes of the same patients and embryos were transferred without a successful pregnancy.

\section{DISCUSSION}

Traditionally, cumulus-enclosed oocytes are overnight co-incubation (18-20 hours) with sperms due to convenient laboratory management during in vitro fertilization. Bungum et al, demonstrated that fertilization could occur as short as $30 \mathrm{sec}$ of co-incubation. ${ }^{7}$ Nagy et al, showed that oocytes were fertilized 2-4 hours after exposure to spermatozoa and the second polar body was emerged approximately $90 \%$ into perivitelline space by 6 hours. ${ }^{8,9}$ Prolonged co-incubation to $18-20$ hours can produce unfavorable environments such as the high concentration of reactive oxygen species which may cause zonal hardening and impair embryonic development and implantation. ${ }^{1}$ Therefore, short coincubation may be an alternative procedure. However, there are inconsistent reports on the outcomes of short and long co-incubation time of gametes. Kattera and Dirnfeld showed that short co-incubation had significant higher implantation and ongoing pregnancy rate., ${ }^{2,3}$ Although, there were varying in co-incubation time, Guo (co-incubation 3 hours), Dai and Xue (co-incubation 4 hours), Liu (co-incubation 5 hours), and Xiong, Chen, 
Zhou and Liu (co-incubation 6 hours) found that implantation rate and clinical pregnancy rate were similar to long co-incubation. ${ }^{4-6,9-13}$ In contradiction, BarraudLange et al, demonstrated that short co-incubation had low numbers of available embryos for transfer. ${ }^{14}$ Nevertheless, a meta-analysis indicates that short coincubation has a significant increase in implantation rate and clinical pregnancy rate. ${ }^{15}$ The authors note that the conclusion may compromise due to differences in study designs and populations. In current study, sibling oocytes were randomly allocated into 2 groups to minimize the confounding factors between patients and the results showed that short co-incubation had no significant difference in fertilization, polyspermy, cleavage of embryos, blastocyst formation, implantation, clinical pregnancy, and live birth rate when compared to long coincubation which go in line with previous studies. ${ }^{4,9-13}$ Therefore, in this context, short co-incubation give no any superior benefit over long co-incubation.

In fact, cumulus cells are necessary for oocyte development and natural fertilization process and may have less benefit during embryonic development as widely known after intracytoplasmic sperm injection (ICSI) which embryo can develop normally without cumulus cells. That means the removal of cumulus cells after short co-incubation might not affect embryonic development. However, early cumulus cell removal can detect fertilization and rescue ICSI may be performed with resulting in higher fertilization rates and optimal embryos compared with rescue ICSI after long coincubation (18 hours). This additional benefit of short coincubation is very interesting. Besides, the time-course of fertilization among early rescue ICSI has a similar pattern to those oocytes that undergo ICSI at the normal time of fertilization and give rise to embryos in a synchronized development with endometrium. ${ }^{9}$ In this study, we removed the cumulus cell at 6 hours and rescue ICSI was conducted in 5 cases of total fertilization failure and resulting in the normal fertilization rate $(79.2 \%)$ which means ICSI after early cumulus cell removal (6 hours) can rescue most of the unfertilized oocytes. Therefore, early rescue ICSI in TFF is an alternative method to alleviate patient from cycle cancellation. Cautiously, rescue ICSI can be potentially injecting fertilized eggs that delayed releasing of the second polar body $(10 \%){ }^{9}$ Theoretically, complete extrusion of the second polar body would be observed by 8 hours after fertilization. ${ }^{9}$ Lui et al, deferred rescue ICSI to 9 hours after insemination to ascertain that fertilized oocytes had already exhibited a sign of fertilization. ${ }^{11}$ Interestingly, polyspermy after rescue ICSI was not increased even performed at 6 hours in our study. Despite the potential benefit of early cumulus cell removal and rescue ICSI, the demand for embryologists to cope with the additional work during the extra period must be taken into account. ${ }^{9}, 11$ Moreover, the cumulus cells are more tuff and difficult for early removal when compared with a later time. A forceful attempt to remove those cumulus cells can damage spindles and microtubules of the oocyte and disturb the integrity of zona pellucida such as a minute fracture which may increase polyspermic fertilization. ${ }^{4,16,17}$ On the other hand, Zhang et al, in a meta-analysis showed that the use of the denuding pipette during cumulus cell removal was not harmful to the clinical pregnancy or implantation rate. ${ }^{15}$ This study found no significant difference in polyspermy, implantation, and clinical pregnancy rate in short and long co-incubation as previous studies. ${ }^{9-13}$

In the short co-incubation and early cumulus cell removal, the patient had a higher premature delivery, twins and low birth weight newborn than long coincubation. ${ }^{5,11-13} \mathrm{Li}$ et al, pointed out that cumulus cell removal at a different time can alter spindle integrity and impair cell division which results in twins or poor fetal growth. ${ }^{18}$ Moreover, inappropriate mechanical stimulation during cumulus cell removal may cause epigenetic change that is related to low birth weight. ${ }^{19}$ Our finding showed that short co-incubation with early cumulus cell removal had comparable obstetric and prenatal outcomes to long co-incubation with late cumulus cell removal.

\section{CONCLUSION}

The present study showed that short co-incubation of gametes had no significant difference in fertilization, polyspermy, embryo development, and pregnancy outcomes when compared to long co-incubation. The short co-incubation with early cumulus cell removal and rescue ICSI may have the potential to help a couple who had total fertilization failure.

\section{ACKNOWLEDGMENTS}

Authors would like to thank Buddhachinaraj Hospital Medical School for providing a professional English reviewer, Mr. Robert Moore, to help edit the manuscript.

Funding: No funding sources

Conflict of interest: None declared

Ethical approval: The study was approved by the Institutional Ethics Committee

\section{REFERENCES}

1. Gianaroli L, Cristina Magli M, Ferraretti AP, Fiorentino A, Tosti E, Panzella S, et al. Reducing the time of sperm oocyte interaction in human in vitro fertilization improves the implantation rate. Hum. Reprod. 1996;11:166-71.

2. Kattera S, Chen C. Short coincubation of gametes in in vitro fertilization improves implantation and pregnancy rates: a prospective, randomized, controlled study. Fertil Steril. 2003;80:1017-21.

3. Dirnfeld M, Shiloh H, Bider D, Harari E, Koifman $\mathrm{M}$, Lahav Baratz $\mathrm{S}$, et al. A prospective randomized controlled study of the effect of short coincubation of 
gametes during insemination on zona pellucida thickness. Gynecol Endocrinol. 2003;17:397-403.

4. Xiong S, Han W, Liu JX, Zhang XD, Liu WW, Liu $\mathrm{H}$, et al. Effects of cumulus cells removal after $6 \mathrm{~h}$ co-incubation of gametes on the outcomes of human IVF. J Assist Reprod Genet. 2011;28:1205-211.

5. Xue Y, Tong X, Jiang L, Zhu H, Yang L, Zhang S. Effect of cumulus cell removal 4hours postinsemination on fertilization and embryo quality: a prospective randomized sibling-oocyte study. J Assist Reprod Genet. 2013;30:1049-53.

6. Dai SJ, Qiao YH, Jin HX, Xin ZM, Su YC, Sun YP, et al. Effect of coincubation time of sperm-oocytes on fertilization, embryonic development, and subsequent pregnancy outcome. System Biol. Reprod Med. 2012;58:348-53.

7. Bungum M, Bungum L, Humaidan P. A prospective study, using sibling oocytes, examining the effect of 30 seconds versus 90 minutes gamete co incubation in IVF. Hum Reprod. 2006;21:518-23.

8. Nagy ZP, Liu J, Joris H, Devroey P, Van Steierteghem A. Time course of oocyte activation, pronucleus formation and cleavage in human oocytes fertilized by intra-cytoplasmic sperm injection. Hum Reprod. 1994;9:1743-8.

9. Chen C, Kattera S. Rescue ICSI of oocytes that failed to extrude the second polar body 6hours postinsemination in conventional IVF. Hum Reprod. 2003; 18:2118-21.

10. Guo N, Yang F, Liu Q, Ren X, Zhao H, Li Y, et al. Effects of cumulus cell removal time during in vitro fertilization on embryo quality and pregnancy outcomes: a prospective randomized sibling-oocyte study. Reprod Biol Endocrinol. 2016;14:18-24.

11. Liu J, Zhang X, Yang Y, Zhao J, Hao D, Zhang J, et al. Long-time vs. short-time insemination of sibling eggs. Exp Ther Med. 2016;12:3756-60.

12. Zhou L, Wang J, Xiao L, Sun H, Wang Y, Geng L, et al. Differential effects of short co-incubation of gametes and early removal of cumulus cells in patients with different fertilizing capabilities. Reprod Bio Med Online. 2016;32:591-6.

13. Liu J, Chen M, Lin C, Weng X, Meng Z, Tang W. Effect of early cumulus cell removal on the fertilization and clinical outcome in human in vitro fertilization. Advance Reprod Sci. 2015;3:50-6.

14. Barraud Lange V, Sifer C, Pocaté K, Ziyyat A, Martin-Pont B, Porcheret R, et al. Short gamete co incubation during in vitro fertilization decreases the fertilization rate and does not improve embryo quality: a prospective auto controlled study. J Assist Reprod Genet. 2008;25:305-10.

15. Zhang XD, Liu JX, Liu WW, Gao Y, Han W, Xiong $\mathrm{S}$, et al. Time of insemination culture and outcomes of in vitro fertilization: a systematic review and meta analysis. Hum Reprod Update. 2013;19:685-95.

16. Payne D, Flaherty SP, Barry MF, Matthews CD. Preliminary observations on polar body extrusion and pronuclear formation in human oocytes using time-lapse video cinematography. Hum Reprod. 1997;12:532-41.

17. Wang WH, Day BN, Wu GM. How does polyspermy happen in mammalian oocytes? Microsc Res Techniq. 2003;61:335-41.

18. Li GP, Bunch TD, White KL, Rickords L, Liu Y, Sessions BR. Denuding and centrifugation of maturing bovine oocytes alters oocyte spindle integrity and the ability of cytoplasm to support parthenogenetic and nuclear transfer embryo development. Mol Reprod Dev. 2006;73:446-51.

19. Kondapalli LA, Perales-Puchalt A. Low birth weight: is it related to assisted reproductive technology or underlying infertility? Fertil Steril. 2013;99:303-10.

Cite this article as: Pongsuthirak P. Comparison of short and long co-incubation time of gametes for in vitro fertilization. Int J Reprod Contracept Obstet Gynecol 2019;8:4366-70. 